OPEN ACCESS

Edited by:

Junguang Tao,

Hebei University of Technology, China

Reviewed by:

Nito Angelo Debacher

Universidade Federal de Santa

Catarina, Brazil

Tao Ji,

Shenzhen University, China

*Correspondence:

Shijie L

lishijie@zjou.edu.cn

Kaibing Xu

xukaibing@dhu.edu.cn

Specialty section:

This article was submitted to Green and Sustainable Chemistry, a section of the journal

Frontiers in Chemistry

Received: 07 August 2018 Accepted: 09 October 2018 Published: 26 October 2018

Citation:

Li S, Jiang W, Xu K, Hu S, LiU Y, Zhou Y and Liu J (2018) Synthesis of Flower-Like Agl/BiOCOOH p-n Heterojunctions With Enhanced Visible-Light Photocatalytic Performance for the Removal of Toxic Pollutants. Front. Chem. 6:518. doi: 10.3389/fchem.2018.00518

\section{Synthesis of Flower-Like} Agl/BiOCOOH p-n Heterojunctions With Enhanced Visible-Light Photocatalytic Performance for the Removal of Toxic Pollutants

\author{
Shijie $\mathrm{Li}^{1 *}$, Wei Jiang ${ }^{1}$, Kaibing X ${ }^{2 *}$, Shiwei Hu ${ }^{1}$, Yu Liu ${ }^{1}$, Yingtang Zhou ${ }^{1}$ and Jianshe $\mathrm{Liu}^{3}$ \\ ${ }^{1}$ Key Laboratory of Key Technical Factors in Zhejiang Seafood Health Hazards, Institute of Innovation and Application, \\ Zhejiang Ocean University, Zhoushan, China, ${ }^{2}$ State Key Laboratory for Modification of Chemical Fibers and Polymer \\ Materials, Research Center for Analysis and Measurement, Donghua University, Shanghai, China, ${ }^{3}$ State Environmental \\ Protection Engineering Center for Pollution Treatment and Control in Textile Industry, College of Environmental Science and \\ Engineering, Donghua University, Shanghai, China
}

In this study, flower-like Agl/BiOCOOH heterojunctions were constructed through a two-step procedure involving the solvothermal synthesis of $\mathrm{BiOCOOH}$ microflowers followed by Agl modification using a precipitation method. These novel photocatalysts were systematically examined by XRD, UV-vis DRS, SEM, TEM, EDS, and PL spectroscopy techniques. The Agl/BiOCOOH heterojunction were studied as a decent photocatalyst for the removal of the industrial dye (rhodamine $\mathrm{B}$, and methyl blue) and antibiotic (tetracycline) under visible light. The $\mathrm{Agl} / \mathrm{BiOCOOH}$ heterojunctions are much more active than bare $\mathrm{BiOCOOH}$, and Agl, which could be ascribed to the improved separation of charge carriers, resulting from the formation of $p-n$ heterojunction between two constituents. The holes $\left(\mathrm{h}^{+}\right)$and superoxide radical $\left(\bullet \mathrm{O}_{2}^{-}\right)$were detected as the main active species responsible for the pollutant degradation. The results showed that a highly efficient visible-light-driven photocatalytic system was developed for the decomposition of toxic pollutants.

Keywords: Agl/BiOCOOH, p-n heterojunction, photocatalysis, dye removal, antibiotic removal

\section{HIGHLIGHTS}

- Novel architectures of Agl nanoparticles anchored on $\mathrm{BiOCOOH}$ micro-flowers were prepared.

- Novel Agl/BiOCOOH p-n heterojunction displayed excellent photocatalytic performance.

- Agl/BiOCOOH heterojunctions significantly enhance the visible-light absorption and boost the charge separation. 


\section{INTRODUCTION}

Harvesting sunlight to degrade industrial pollutants in wastewater is an effective and sustainable technology for environmental purification (Chong et al., 2010; Zhu and Wang, 2017). The key of the photocatalysis technique is to develop catalysts with superior photocatalytic performance (Zhang et al., 2015; Ji et al., 2017; Wang and Astruc, 2017; Zhu and Wang, 2017; Moroz et al., 2018; Wu et al., 2018). Till now, various photocatalysts have been constructed and studied (Zhang et al., 2012, 2016; Wang and Astruc, 2017; Mousavi et al., 2018). Among them, Bi-based semiconductors have been regarded as excellent photocatalysts by virtue of its decent photocatalytic activity, strong photo-redox driving force, high chemical stability and nontoxicity (Ahern et al., 2015). However, superior photoredox force is commonly accompanied with a wide band gap $\left(E_{\mathrm{g}}\right)$, which substantially restrains the visible-light absorption. Therefore, the enhancement of visible-light absorption of $\mathrm{Bi}$ based photocatalysts is quite of necessity (Li et al., 2018a,b; Lin et al., 2018).

Various strategies have been applied to ameliorate the visible-light response of Bi-based semiconductors through metal/nonmetal doping (Wu et al., 2017; Li et al., 2018d,e), architecture tailoring (Xiong et al., 2011; Li et al., 2016), heterojunction constructing (Li et al., 2018c; Zhang and Ma, 2018). In particular, the combination of Bi-based semiconductors with narrow band gap semiconductors is an effective way to improve the photocatalytic performance, due to the improved visible-light absorption and boosted separation of electron-hole pairs at the interface (Li et al., 2017d).

$\mathrm{BiOCOOH}$, as a type of layered Bi-based oxides, is composed of $\left[\mathrm{Bi}_{2} \mathrm{O}_{2}\right]^{2+}$ fluorite-like layers intercalating by formic acid (Wei et al., 2015; Xu et al., 2016, 2018; Chen et al., 2018). However, the wide band gap $\left(E_{\mathrm{g}}=3.4 \mathrm{eV}\right)$ of $\mathrm{BiOCOOH}$ makes it only active under ultraviolet light irradiation, which severely limits its photocatalytic performance (Xu et al., 2018). The construction of $\mathrm{BiOCOOH}$-based heterojunctions is advantageous for improving the visible-light photocatalytic performance of BiOCOOH (Chai and Wang, 2015; Chen et al., 2016). As a result, some BiOCOOHbased heterojunctions have been constructed and exhibit better photocatalytic activity under visible light (Chai and Wang, 2015; Li et al., 2017e,f; Chen et al., 2018). The further exploration of novel $\mathrm{BiOCOOH}$-based heterojunction catalysts is still required to provide more potential candidates for practical application and to reveal the reasons for the synergy effect between the constituents.

Recently, AgI has proved a good photosensitizer due to the fact that its narrow band gap $(\sim 2.7 \mathrm{~V})$ is in favor of the light absorption of semiconductors (Wang et al., 2016; Liang et al., 2017, 2018; Xiang et al., 2018). Hence, it inspires us to combine $\mathrm{BiOCOOH}$ with AgI for achieving novel AgI/BiOCOOH p-n heterojunctions with distinguished visible-light photocatalytic performance.

In this study, we have successfully fabricated flower-like $\mathrm{AgI} / \mathrm{BiOCOOH}$ heterojucntions for the first time, through a simple precipitation approach at room temperature. Substantial improvement of the visible-light harvesting ability can be observed after the introduction of AgI. Meanwhile, the novel p-n heterojunction between AgI and $\mathrm{BiOCOOH}$ ensures the effective separation of photo-generated electron and holes. As a result, the $\mathrm{AgI} / \mathrm{BiOCOOH}$ heterojucntions exhibited much higher activity in the photocatalytic degradation of rhodamine $\mathrm{B}(\mathrm{RhB})$, and methyl blue (MB), and tetracycline hydrochloride (TC) antibiotic under visible light. This work offers a simple way to improve the photocatalytic activity of $\mathrm{BiOCOOH}$ toward pollutant removal by coupling with AgI.

\section{EXPERIMENT}

\section{Chemicals}

$\mathrm{Bi}\left(\mathrm{NO}_{3}\right)_{3} \bullet 5 \mathrm{H}_{2} \mathrm{O}$, glycerol, N,N-dimethyformamide (DMF), methyl blue (MB), tetracycline hydrochloride (TC), rhodamine $\mathrm{B}$ (RhB), $\mathrm{AgNO}_{3}, \mathrm{KI}$, iso-propanol (IPA), ammonium oxalate $(\mathrm{AO})$, and $p$-benzoquinone (BQ) were bought from Chemical Reagent factory (China).

\section{Synthesis}

$\mathrm{BiOCOOH}$ microspheres were prepared via a solvothermal method. Briefly, $\mathrm{Bi}\left(\mathrm{NO}_{3}\right)_{3} \bullet 5 \mathrm{H}_{2} \mathrm{O}(4 \mathrm{mmol})$ was dissolved in the solution of $50 \mathrm{~mL}$ of glycerol, $20 \mathrm{~mL}$ of $\mathrm{DMF}$ and $10 \mathrm{~mL}$ of $\mathrm{H}_{2} \mathrm{O}$ with the assistance of ultra-sonication. After being stirring for $1 \mathrm{~h}$, the solution was poured into a $100 \mathrm{~mL}$ autoclave and kept at $160^{\circ} \mathrm{C}$ for $20 \mathrm{~h}$. When the reaction finished and the system was cooled down, the white precipitants separated from the suspension were washed with deionized water and ethanol for several times, and dried at $70^{\circ} \mathrm{C}$ overnight.

Flower-like $\mathrm{AgI} / \mathrm{BiOCOOH}$ heterojunctions were fabricated by a simple in situ precipitation method at room temperature. Firstly, $1 \mathrm{mmol} \mathrm{BiOCOOH}$ was dispersed in $50 \mathrm{~mL}$ of deionized water containing $0.1 \mathrm{mmol} \mathrm{AgNO}_{3}$ to form a

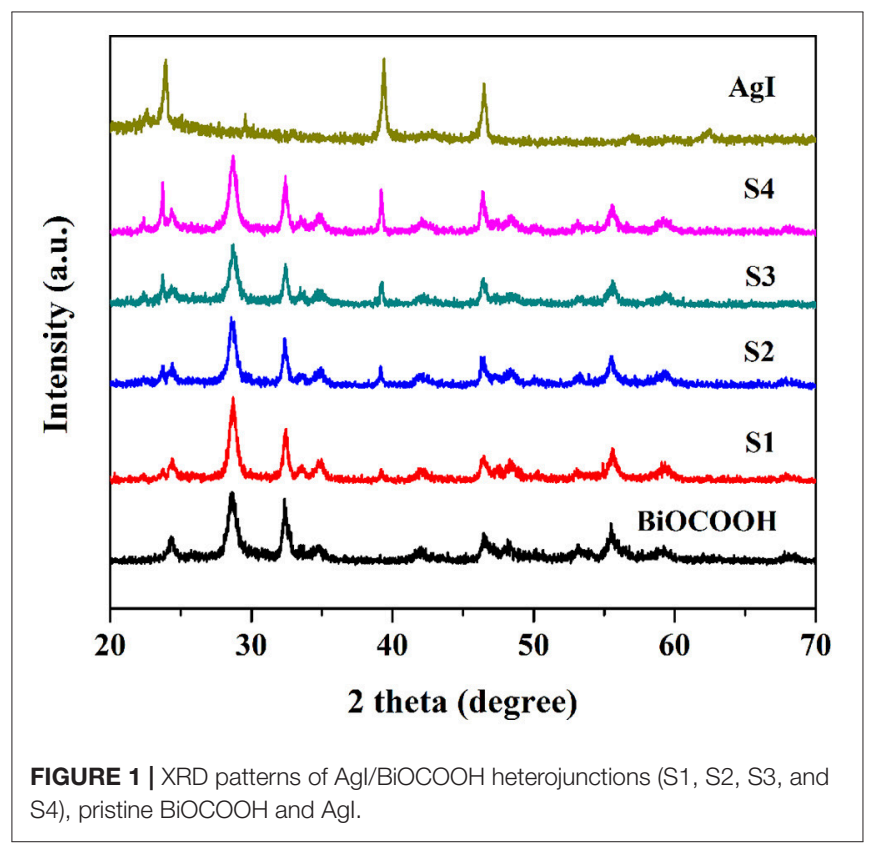



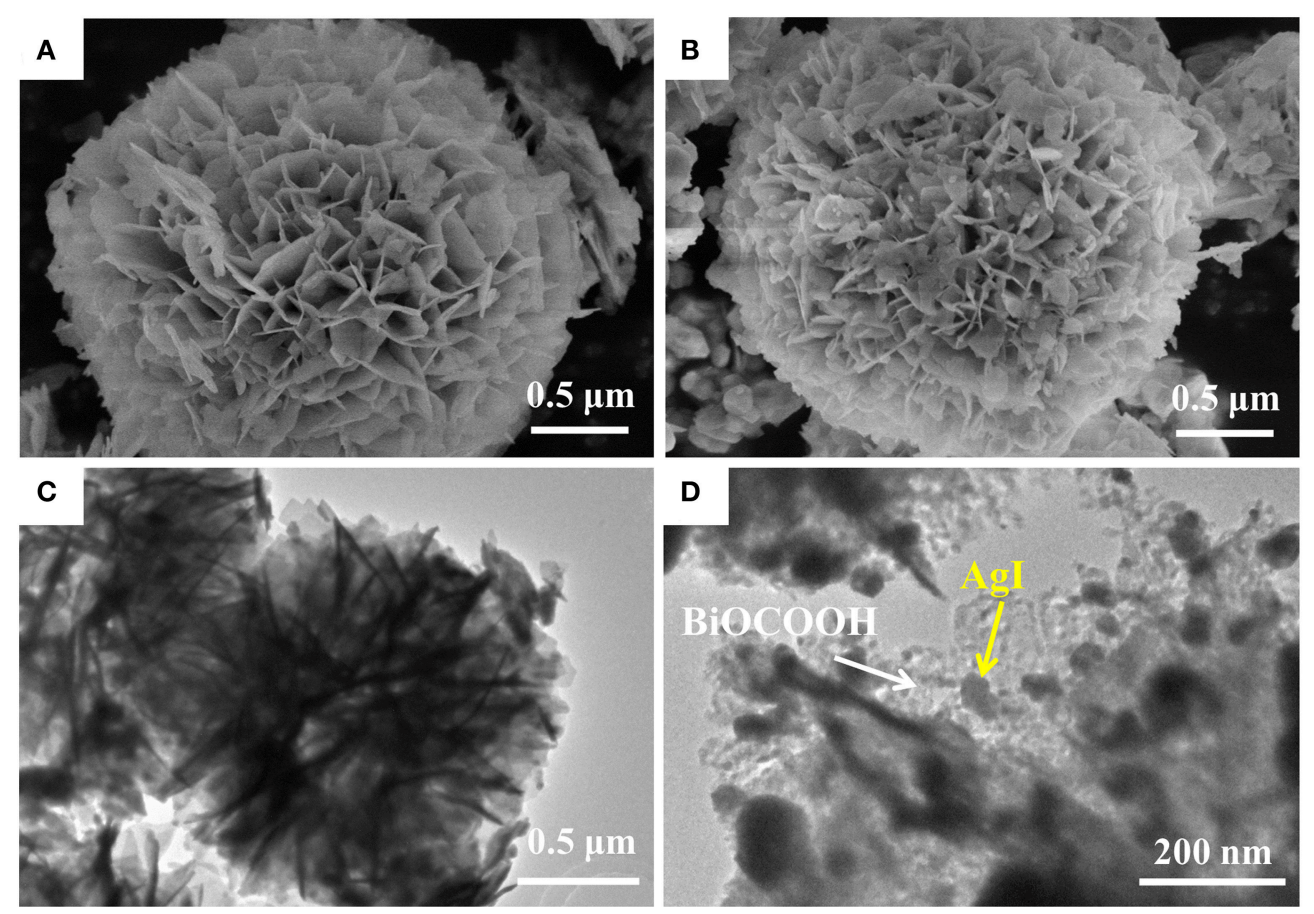

FIGURE 2 | SEM images of bare BiOCOOH (A) and S3 (B); TEM images of S3 (C,D)

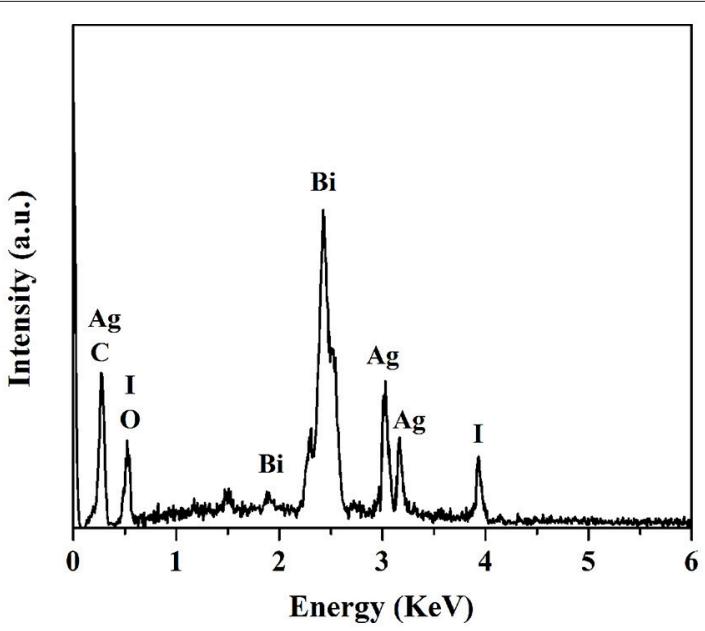

FIGURE 3 | EDS spectra of S3.

homogeneous suspension and kept stirring for $2 \mathrm{~h}$ in darkness. Secondly, $10 \mathrm{~mL}$ of $\mathrm{KI}$ solution $(0.01 \mathrm{M})$ was added into the above suspension slowly under continuously stirring in darkness. After being stirring for $3 \mathrm{~h}$, the obtained solid named as S1 was washed, and dried at $70^{\circ} \mathrm{C}$ overnight. By varying the addition amount of $\mathrm{AgNO}_{3}$ and $\mathrm{KI}$, the other heterojunctions with various $\mathrm{AgI} / \mathrm{BiOCOOH}$ molar ratios (0.5/1, $1 / 1$, and $1.5 / 1$ ) were fabricated and named as S2, S3, and S4, respectively.

\section{Characterization}

$\mathrm{X}$-ray diffraction (XRD) patterns of the samples were collected on a Bruker D8 Advance diffractometer with $\mathrm{Cu} \mathrm{K} \alpha$ radiation. The scanned range of $2 \theta$ was from $20^{\circ}$ to $80^{\circ}$. Hitachi S -4800 scanning electron microscope (SEM), and energy dispersive Xray spectroscopy (EDX), and JEM-2010F transmission electron microscope were used to investigate the microstructures and compositions of the samples. The UV-vis diffused reflectance spectra (DRS) were recorded on a Shimadzu UV-2600 UV-vis spectrophotometer. The photoluminescence (PL) spectra were recorded on a Hitachi F-7000 fluorescence spectrophotometer. The Brunauer-Emmett-Teller (BET) surface areas were determined by a Micromeritics ASAP 2020 equipment.

\section{Photocatalytic Tests}

The photocatalytic degradation of $\mathrm{RhB}, \mathrm{MB}$, or TC was executed to evaluate the activity of catalysts. Typically, $30 \mathrm{mg}$ of catalyst was first added into $100 \mathrm{~mL}$ of $\mathrm{RhB}\left(10 \mathrm{mg} \mathrm{L}^{-1}\right), \mathrm{MB}\left(10 \mathrm{mg} \mathrm{L}^{-1}\right)$, or TC $\left(20 \mathrm{mg} \mathrm{L}^{-1}\right)$ solution in a reactor and then stirred for half an hour in the dark. Subsequently, the photocatalytic reaction was initiated when a $300 \mathrm{~W}$ xenon lamp coupled with a UV-cutoff filter $(\lambda>400 \mathrm{~nm})$ switched on, $2 \mathrm{~mL}$ of the suspensions were extracted at certain intervals, and separated by centrifugation to get the supernatant solutions. The concentrations of solutions were determined by using a UV -2600 spectrophotometer. Total organic carbon (TOC) experiments were conducted by the photocatalytic degradation of $\mathrm{RhB}\left(40 \mathrm{mg} \mathrm{L}^{-1}, 150 \mathrm{~mL}\right)$ solution over $200 \mathrm{mg}$ of S3. 

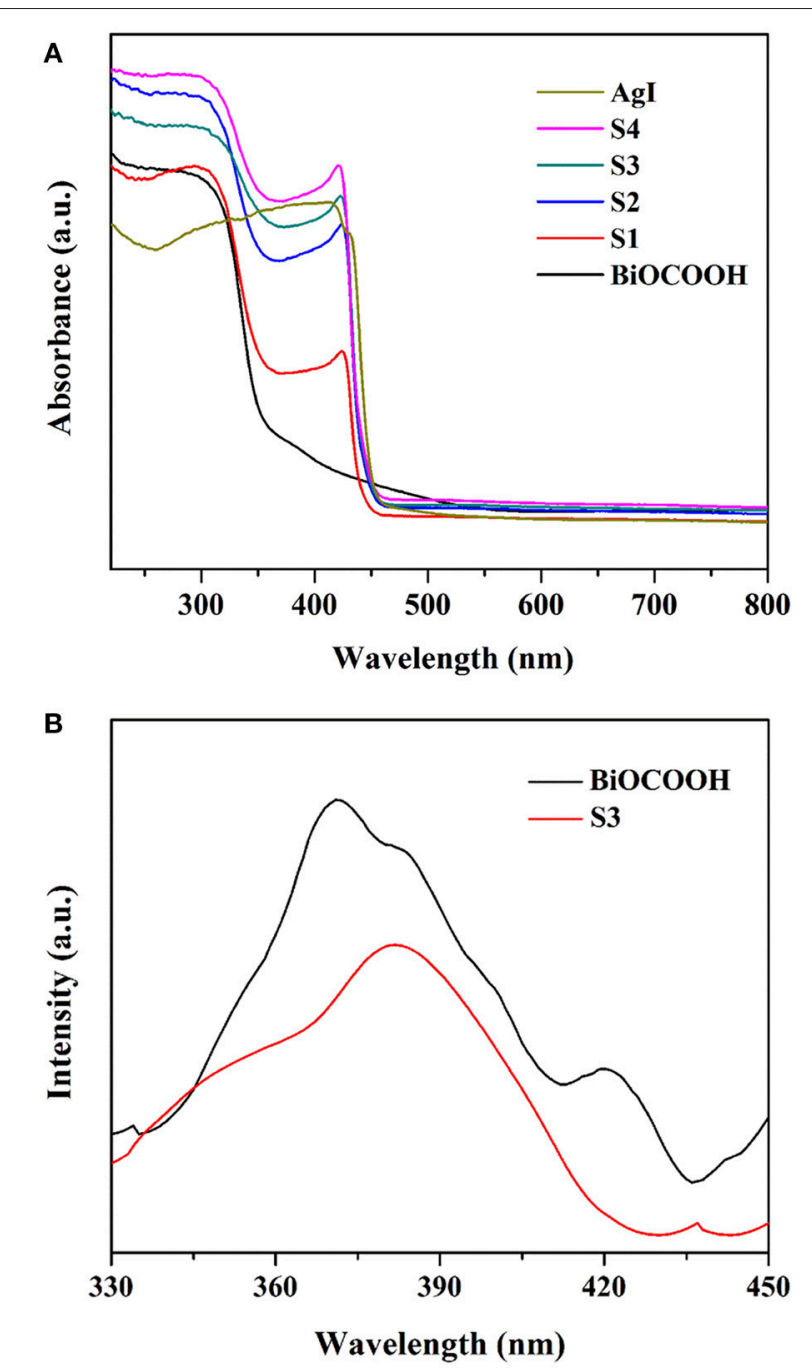

FIGURE 4 | (A) UV-Vis DRS of bare $\mathrm{BiOCOOH}$, Agl, and Agl/BiOCOOH heterojunctions (S1, S2, S3, and S4). (B) PL spectra of pristine $\mathrm{BiOCOOH}$ and S3 with an excitation wavelength of $300 \mathrm{~nm}$.

\section{RESULTS AND DISCUSSION}

\section{Characterization}

A series of $\mathrm{AgI} / \mathrm{BiOCOOH}$ heterojunctions with different molar ratios $(0.1 / 1,0.5 / 1,1 / 1$, and $1.5 / 1)$ were prepared and denoted as S1, S2, S3, and S4, respectively. AgI, BiOCOOH, and $\mathrm{AgI} / \mathrm{BiOCOOH}$ heterojunctions (S1, S2, S3, and S4) were characterized by XRD and the XRD patterns are shown in Figure 1. Pure AgI and $\mathrm{BiOCOOH}$ could be indexed as the miersite AgI (JCPDS 78-0641) and the tetragonal $\mathrm{BiOCOOH}$ (JCPDS 35-0939), respectively. The XRD patterns of $\mathrm{AgI} / \mathrm{BiOCOOH}$ heterojunctions generally resemble that of $\mathrm{BiOCOOH}$, but some peaks belonging to AgI are also detected, indicating the co-existence of $\mathrm{AgI}$ and $\mathrm{BiOCOOH}$ in these heterojunctions.

The microstructure and morphology of $\mathrm{BiOCOOH}$ and the $\mathrm{AgI} / \mathrm{BiOCOOH}$ heterojunction were surveyed by SEM and TEM
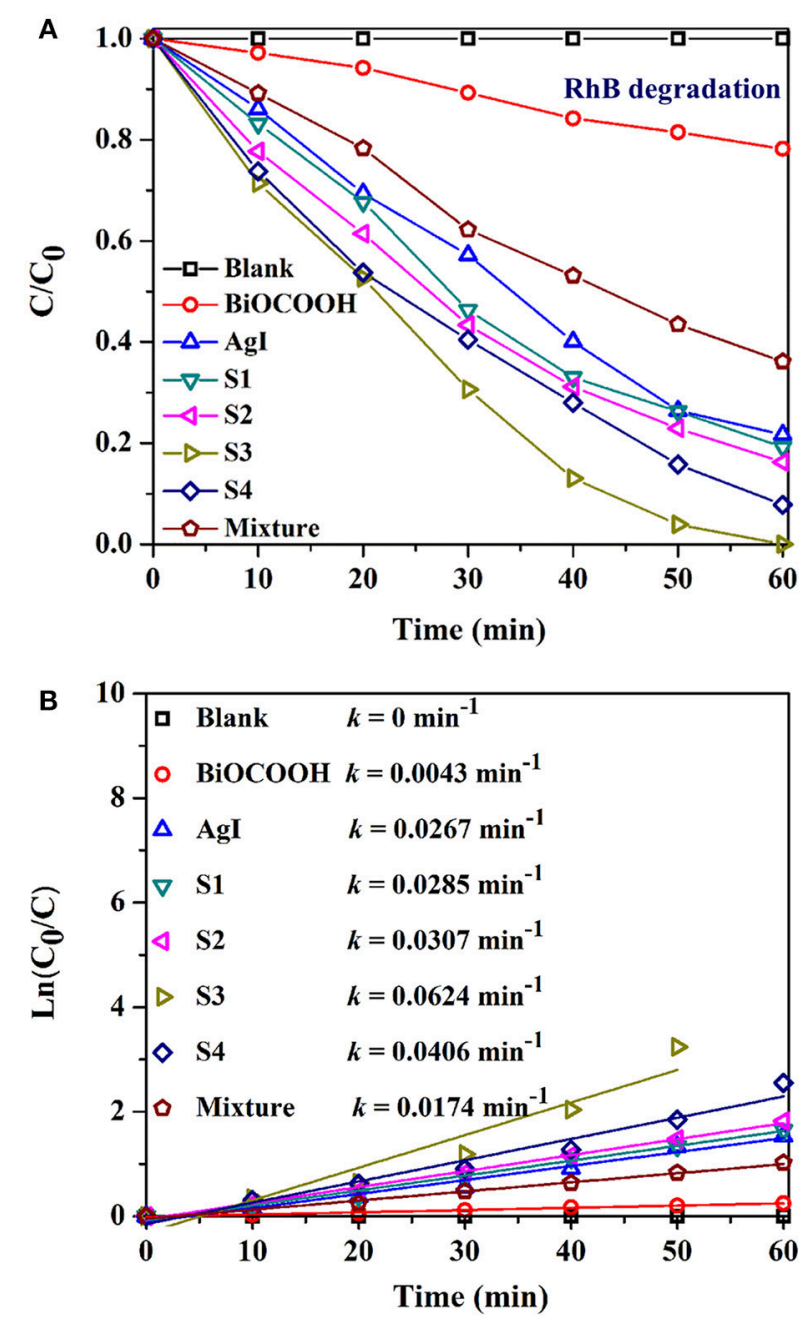

FIGURE 5 | (A) Photocatalytic degradation of RhB over various catalysts under visible light. (B) The kinetic curves over different catalysts.

(Figure 2). Obviously, bare $\mathrm{BiOCOOH}$ exhibits hierarchical structures assembled from numerous nano-sheets with size of 150-500 nm (Figure 2A). After the introduction of AgI, the asprepared $\mathrm{AgI} / \mathrm{BiOCOOH}$ maintained the microsphere structure. Representatively, S3 consists of flower-like $\mathrm{BiOCOOH}$ spheres and AgI nanoparticles (size: 20-150 nm, Figure 2B), in which AgI nanoparticles are deposited on $\mathrm{BiOCOOH}$ spheres. The detailed structural information was further obtained by the TEM images. Figures 2C,D confirms that AgI nanoparticles and $\mathrm{BiOCOOH}$ spheres are attached to each other, indicating the successful preparation of $\mathrm{AgI} / \mathrm{BiOCOOH}$ heterojunction with a well contacted interface. Additionally, the energy dispersive spectroscopy (EDS) spectra reveals the presence of chemical elements Ag, I, C, O, and Bi in S3 (Figure 3).

For photocatalysts, the optical absorption behavior is recognized as one of the most significant factors in determining their photocatalytic activity (Akhundi and Habibi-Yangjeh, 2017; Li et al., 2017c; Wang et al., 2018). Thus, the optical 


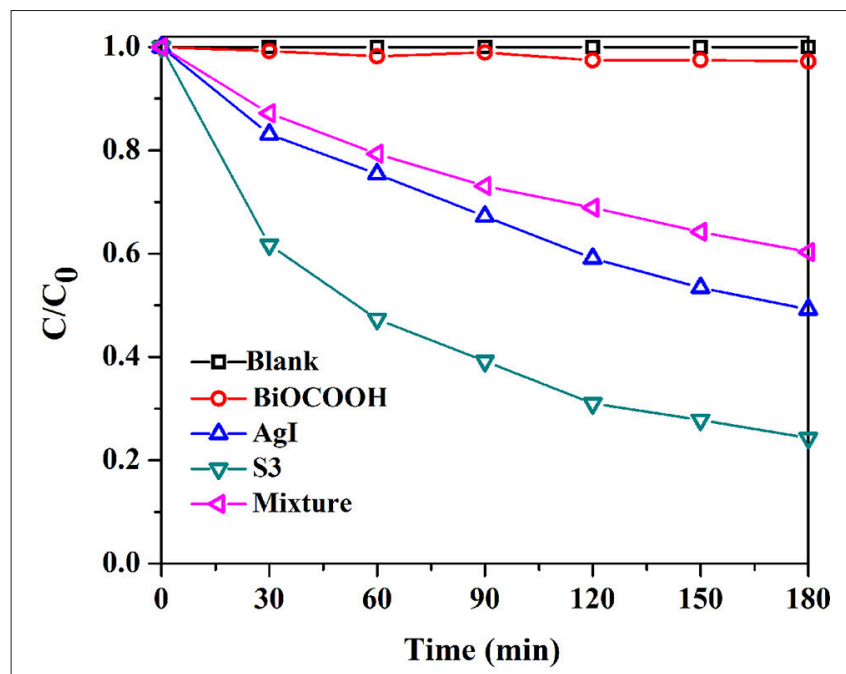

FIGURE 6 | The TC degradation curves over various catalysts under visible light.

absorption properties of $\mathrm{BiOCOOH}, \mathrm{AgI}$ and the $\mathrm{AgI} / \mathrm{BiOCOOH}$ heterojunctions were surveyed by UV-Vis diffuse reflection spectra (UV-Vis DRS) and the results are presented in Figure 4A. The absorption edges of pure AgI and $\mathrm{BiOCOOH}$ are about $450 \mathrm{~nm}\left(E_{\mathrm{g}}=2.76 \mathrm{eV}\right)$ (Wang et al., 2016; Liang et al., 2017) and $365 \mathrm{~nm}\left(E_{\mathrm{g}}=3.40 \mathrm{eV}\right)$ (Chai and Wang, 2015; Li et al., 2017f), respectively, in accordance with the reported results (Chai and Wang, 2015; Li et al., 2017f). Compared to pure $\mathrm{BiOCOOH}, \mathrm{AgI} / \mathrm{BiOCOOH}$ heterojunctions are endowed with intensive visible-light absorption.

The band potential of AgI and $\mathrm{BiOCOOH}$ could be calculated by the following equations:

$$
\begin{gathered}
E_{\mathrm{VB}}=X-E_{0}+0.5 E_{\mathrm{g}} \\
E_{\mathrm{CB}}=E_{\mathrm{VB}}-E_{\mathrm{g}}
\end{gathered}
$$

Where, the $X$ represents the absolute electronegativity of the semiconductor. $E_{0}$ value is $\sim 4.5 \mathrm{eV}$. $E_{\mathrm{g}}$ means the band gap of the semiconductor. Hence, the $E_{\mathrm{CB}}$ and $E_{\mathrm{VB}}$ of $\mathrm{AgI}$ are calculated to be -0.40 and $2.36 \mathrm{eV}$, and those of $\mathrm{BiOCOOH}$ are -0.67 and $2.73 \mathrm{eV}$.

The separation efficiency of charge carriers is strongly related to the photocatalytic performance of the catalyst ( $\mathrm{Li}$ et al., 2017a; Zhang et al., 2017). Thus, the PL technique was employed to probe the separation efficiency of electrons and holes. As shown in Figure 4B, BiOCOOH displays a main emission peak centered at ca. $370 \mathrm{~nm}$. Intriguingly, S3 exhibits a much lower PL intensity than pristine $\mathrm{BiOCOOH}$, illustrating that the formation of $\mathrm{AgI} / \mathrm{BiOCOOH}$ heterojunction favors the separation of photoinduced electrons and holes.
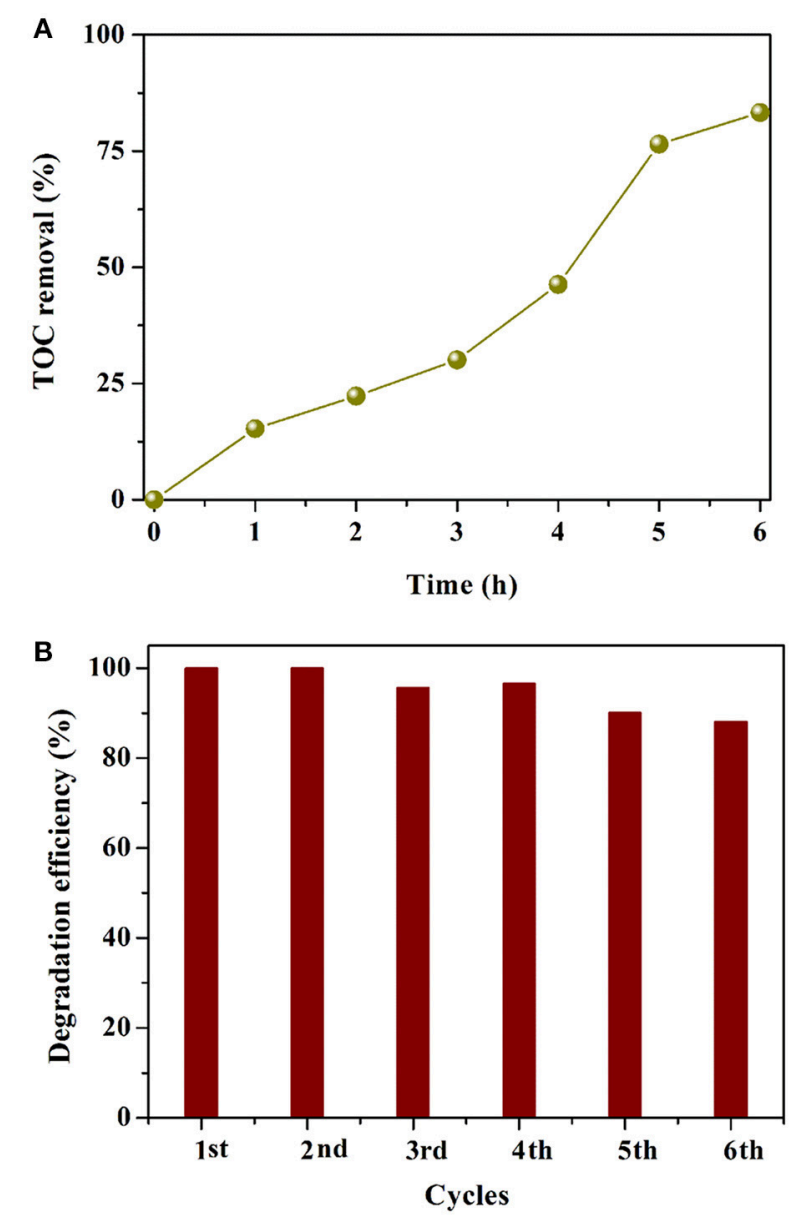

FIGURE 7 | (A) TOC removal curves in the presence of S3. (B) Cycling runs in the degradation of RhB over S3.

\section{Photocatalytic Property}

The photocatalytic activity of the $\mathrm{AgI} / \mathrm{BiOCOOH}$ heterojunctions were tested through the degradation of industrial dyes (RhB, and $\mathrm{MB}$ ), and antibiotic (TC) under visible light. Figure 5A shows the $\mathrm{RhB}$ degradation curves. No RhB degradation happened in the absence of catalysts. Only 21.8 or $78.3 \%$ of $\mathrm{RhB}$ degradation could be reached in 60 min when pristine $\mathrm{BiOCOOH}$ or $\mathrm{AgI}$ presented. Intriguingly, a synergistic effect is found between $\mathrm{AgI}$ and $\mathrm{BiOCOOH}$ when they are combined, endowing these heterojunctions with higher activity than that of pristine AgI or $\mathrm{BiOCOOH}$. Among these AgI/BiOCOOH heterojunctions, S3 showed the best photocatalytic activity with $100 \%$ of $\mathrm{RhB}$ degraded within $60 \mathrm{~min}$. Of note, the molar ratio of AgI to BiOCOOH plays a crucial role in the photocatalytic activity of the heterojunction. A much higher or lower molar ratio is not favorable for improving the activity of these heterojunctions. Besides, the BET surface areas of the catalysts were investigated and the results were listed in Table S1. It can be seen that the BET surface areas of BiOCOOH, S1, S2, S3, and S4 are 26.13, 28.27, 24.26, 20.72, and $15.21 \mathrm{~m}^{2} \mathrm{~g}^{-1}$, respectively. Though $\mathrm{S} 3$ shows the highest activity, 
its BET surface area is not the largest among these samples. Obviously, the surface area does not play a dominant role in determining the photocatalytic performance of $\mathrm{AgI} / \mathrm{BiOCOOH}$.

To further explore the synergistic effect, the photocatalytic activity of the physical mixture (46.5 wt $\%$ AgI +53.5 wt $\%$ $\mathrm{BiOCOOH}$ ) was also measured and the $\mathrm{RhB}$ degradation efficiency in $60 \mathrm{~min}$ is much lower than that by using S3 as the catalyst, demonstrating that the establishment of $p-n$ heterojunction favors the activity enhancement.

The kinetic behaviors of the $\mathrm{RhB}$ degradations in the presence of various catalysts also have been studied. The $\mathrm{RhB}$ degradation process can be fitted well by the pseudo-first-order model. As shown in Figure 5B, S3 achieves the highest $\mathrm{k}$ value of 0.0624 $\mathrm{min}^{-1}, 13.5,1.3$, or 2.6 folds greater than that of bare $\mathrm{BiOCOOH}$ $\left(0.0043 \mathrm{~min}^{-1}\right)$, AgI $\left(0.0267 \mathrm{~min}^{-1}\right)$, or the mixture $(0.0174$ $\left.\min ^{-1}\right)$.

MB dye, and TC antibiotic, two types of toxic pollutants were also employed to further assess the photocatalytic performance of $\mathrm{AgI} / \mathrm{BiOCOOH}$ (Figure 6, Figure S1). Attractively, the MB

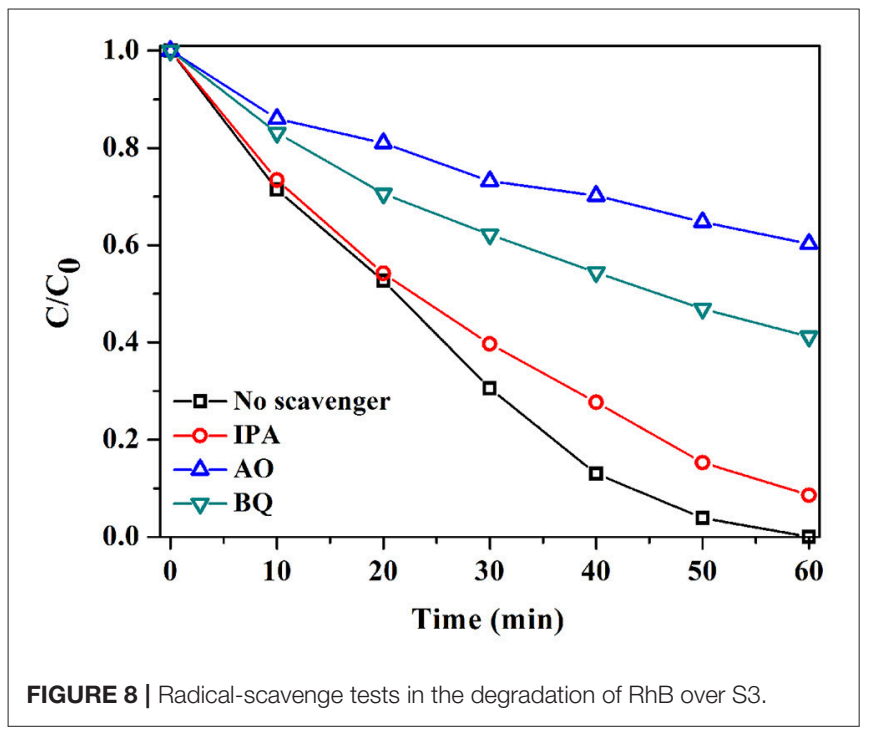

degradation efficiency achieved by using S3 reaches $85.2 \%$ in 90 min of reaction, much better than that achieved by employing pure AgI (68.7\%), BiOCOOH (32.8\%), or a physical mixture $(60.2 \%)$ as the catalyst. In addition, a similar phenomenon is found when employing TC (Figure 6) as a target pollutant. The optimized S3 showed an excellent photocatalytic activity with TC degradation efficiency of $75.7 \%$ in $3 \mathrm{~h}$, considerably higher than those of bare $\mathrm{BiOCOOH}(2.8 \%)$ and $\mathrm{AgI}(50.7 \%)$ as well as their mixtures (39.6\%).

To assess the mineralization ability of $\mathrm{AgI} / \mathrm{BiOCOOH}$, the TOC concentration during $\mathrm{RhB}\left(150 \mathrm{~mL}, 40 \mathrm{mg} \mathrm{L}^{-1}\right)$ degradation by S3 (200 mg) was measured and analyzed. As illustrated in Figure 7A, notably, the TOC removal efficiency increases with the prolonging of reaction time and the final TOC mineralization efficiency yields $83.4 \%$ in $6 \mathrm{~h}$, suggesting that $\mathrm{AgI} / \mathrm{BiOCOOH}$ has the great potential for the deep treatment of toxic pollutants.

The stability of AgI/BiOCOOH (S3) was tested via recycling for six runs in the degradation of $\mathrm{RhB}$. As depicted in Figure $7 \mathbf{B}$, the $\mathrm{RhB}$ degradation efficiency at $60 \mathrm{~min}$ of the first run is $100 \%$, while that value becomes $88.1 \%$ in the sixth run. The slight decline in the activity may be due to the catalyst loss in the recycling experiments. Besides, a small amount of $\mathrm{Ag}^{0}$ was produced after the cycling tests, which has been proved by the XRD results (Figure S2). It has been demonstrated that Ag/AgI displays a stable structure, which can facilitate the separation of charge carriers (Ren et al., 2018; Yuan et al., 2018). These results verify that $\mathrm{AgI} / \mathrm{BiOCOOH}(\mathrm{S} 3)$ possesses good stability.

\section{The Photocatalytic Mechanism}

Radicals generated during the photocatalytic reaction are mainly involved in the degradation of pollutant (Wang et al., 2011). Therefore, to figure out their role in the degradation of $\mathrm{RhB}$, radical-scavenge experiments were performed. As Figure 8 reveals, with the introduction of isopropanol (IPA), only a slight decrease in the activity of S3 was observed, with a $\mathrm{RhB}$ degradation efficiency of $99.14 \%$. The result signified that $\bullet \mathrm{OH}$ showed a negligible effect on $\mathrm{RhB}$ degradation. Instead, the addition of benzoquinone (BQ) and ammonium oxalate (AO) substantially inhibited the degradation of RhB,

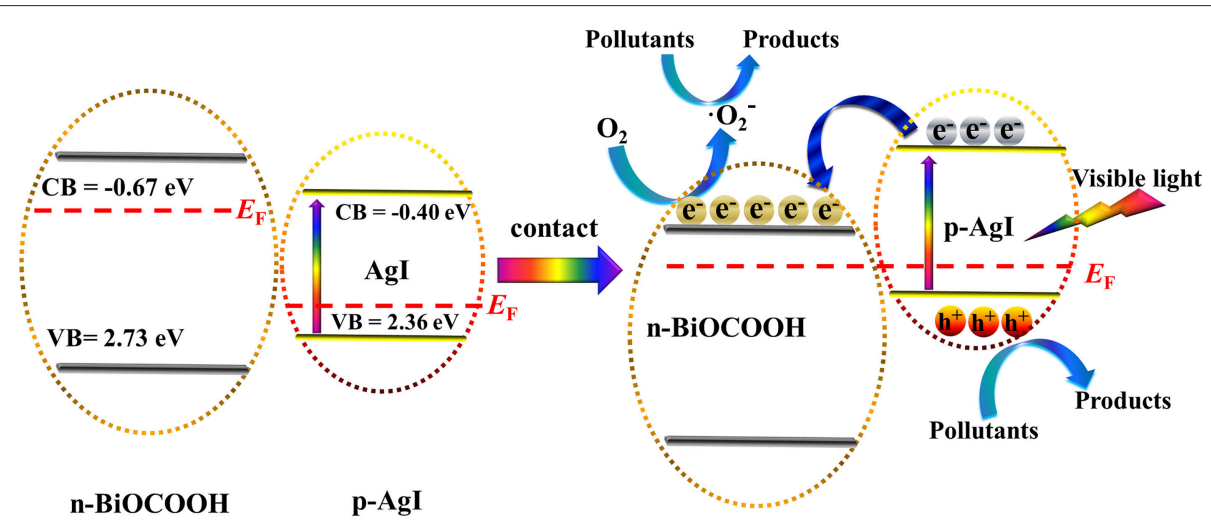

FIGURE 9 | Proposed mechanism of pollutant degradation over Agl/BiOCOOH under visible light. 
with RhB degradation efficiencies of only 58.8 and $39.7 \%$. The results demonstrated the significant roles of $\bullet \mathrm{O}_{2}^{-}$and $\mathrm{h}^{+}$in photocatalytic degradation of $\mathrm{RhB}$ over $\mathrm{AgI} / \mathrm{BiOCOOH}$.

Based on the above analysis, a possible mechanism for the degradation of pollutants over $\mathrm{AgI} / \mathrm{BiOCOOH}$ under visible light is brought forward as outlined in Figure 9. As shown in Figure 9, the Fermi level $\left(E_{\mathrm{F}}\right)$ and conduction band $(\mathrm{CB})$ of $p$-type AgI are lower than those of $n$-type $\mathrm{BiOCOOH}$ before they are in contact. After the contact between $p$-type AgI and $n$-type $\mathrm{BiOCOOH}$, the $E_{\mathrm{F}}$ of AgI shifts upward, while that of $\mathrm{BiOCOOH}$ shifts downward until the $E_{\mathrm{F}}$ of both semiconductors achieves an equilibrium. Correspondingly, the CB and VB of AgI moves up along with the $E_{\mathrm{F}}$, and those of $\mathrm{BiOCOOH}$ moves down. As a consequence, the $\mathrm{CB}$ and $\mathrm{VB}$ of $\mathrm{BiOCOOH}$ are lower than those of AgI. Similar phenomenon was also observed in other p-n heterojunction photocatalysts (Li et al., 2017b). Under visible-light irradiation, AgI is excited to produce photogenerated electrons and holes on the $\mathrm{CB}$ and VB. The photogenerated electrons on the $\mathrm{CB}$ of AgI can easily drift to the $\mathrm{CB}$ of $\mathrm{BiOCOOH}$, which is beneficial to the separation of electrons and holes (Ye et al., 2018), as proved by the PL results (Figure 4B). Subsequently, the electrons enriched on the $\mathrm{CB}$ of $\mathrm{BiOCOOH}$ can be captured by $\mathrm{O}_{2}$ to yield $\bullet \mathrm{O}_{2}^{-}$radical. Finally, the produced $-\mathrm{O}_{2}^{-}$and $\mathrm{h}^{+}$reactive species are involved in the degradation of toxic pollutants (RhB/MB/TC). Apparently, the construction of p-n heterojunction between $p$-type $\mathrm{AgI}$ and $n$-type $\mathrm{BiOCOOH}$ is favorable for the separation of electron-hole pairs, further enhancing the photocatalytic performance.

\section{CONCLUSIONS}

AgI nanoparticles interspersed-BiOCOOH hetero-structures were prepared by a simple method. The visible-light harvesting ability is substantially improved with the introduction of AgI,

\section{REFERENCES}

Ahern, J. C., Fairchild, R., Thomas, J. S., Carr, J., and Patterson, H. H. (2015). Characterization of BiOX compounds as photocatalysts for the degradation of pharmaceuticals in water. Appl. Catal. B-Environ. 179, 229-238. doi: 10.1016/j.apcatb.2015.04.025

Akhundi, A., and Habibi-Yangjeh, A. (2017). Graphitic carbon nitride nanosheets decorated with $\mathrm{CuCr}_{2} \mathrm{O}_{4}$ nanoparticles: novel photocatalysts with high performances in visible light degradation of water pollutants. J. Colloid Interface Sci. 504, 697-710. doi: 10.1016/j.jcis.2017.06.025

Chai, B., and Wang, X. (2015). Enhanced visible light photocatalytic activity of $\mathrm{BiOI} / \mathrm{BiOCOOH}$ composites synthesized via ion exchange strategy. RSC Adv. 5, 7589-7596. doi: 10.1039/C4RA10999F

Chen, L., He, J., Liu, Y., Chen, P., Au, C.-T., and Yin, S.-F. (2016). Recent advances in bismuth-containing photocatalysts with heterojunctions. Chin. J. Catal. 37, 780-791. doi: 10.1016/S1872-2067(15)61061-0

Chen, P., Zhang, Q. X., Su, Y. H., Shen, L. Z., Wang, F. L., Liu, H. J., et al. (2018). Accelerated photocatalytic degradation of diclofenac by a novel CQDs/BiOCOOH hybrid material under visible-light irradiation: dechloridation, detoxicity, and a new superoxide radical model study. Chem. Eng. J. 332, 737-748. doi: 10.1016/j.cej.2017.09.118

Chong, M. N., Jin, B., Chow, C. W. K., and Saint, C. (2010). Recent developments in photocatalytic water treatment technology: a review. Water Res. 44, 2997-3027. doi: 10.1016/j.watres.2010.02.039 compared with pristine $\mathrm{BiOCOOH}$. In the photocatalytic pollutant ( $\mathrm{RhB}, \mathrm{MB}$, and $\mathrm{TC}$ ) degradation, the $\mathrm{AgI} / \mathrm{BiOCOOH}$ heterojunctions, especially S3, displayed much higher photocatalytic activity than pristine $\mathrm{BiOCOOH}$ and AgI. Moreover, S3 possesses good reusability. The synergy effect on photocatalytic performance can be ascribed to the $p-n$ heterostructure with improving visible-light absorption and promoting the separation of electron and holes. This work indicates that the well-designed $\mathrm{AgI} / \mathrm{BiOCOOH}$ heterojunction could be potentially employed to remedy environment.

\section{AUTHOR CONTRIBUTIONS}

SL designed and carried out the experiments, and analyze the data. SH, WJ, YL, KX, YZ, and JL assisted with part of the experiments. SL finished the manuscript. All authors have read and agreed the submission of the manuscript.

\section{FUNDING}

This work has been financially supported by the National Natural Science Foundation of China (51708504, 31501573 and 51602049), the Public Projects of Zhejiang Province (LGN18E080003), the Science and Technology Project of Zhoushan (2017C41006, 2016C41012, 2015C21014, and 2015C21013), the National Key Research Development Program of China (2016YFC0400501) and the Start-up Research Funding from Zhejiang Ocean University (12215090117).

\section{SUPPLEMENTARY MATERIAL}

The Supplementary Material for this article can be found online at: https://www.frontiersin.org/articles/10.3389/fchem. 2018.00518/full\#supplementary-material
Ji, T., Cui, Z., Zhang, W., Cao, Y., Zhang, Y., He, S.-A., et al. (2017). UV and visible light synergetic photodegradation using rutile $\mathrm{TiO}_{2}$ nanorod arrays based on a p-n junction. Dalton Trans. 46, 4296-4302. doi: 10.1039/C7DT00261K

Li, S., Hu, S., Jiang, W., Liu, Y., Liu, Y., Zhou, Y., et al. (2018a). $\mathrm{Ag}_{3} \mathrm{VO}_{4}$ nanoparticles decorated $\mathrm{Bi}_{2} \mathrm{O}_{2} \mathrm{CO}_{3}$ micro-flowers: an efficient visible-lightdriven photocatalyst for the removal of toxic contaminants. Front. Chem. 6:255. doi: $10.3389 /$ fchem. 2018.00255

Li, S., Hu, S., Jiang, W., Zhou, Y., Liu, J., and Wang, Z. (2018b). Facile synthesis of cerium oxide nanoparticles decorated flower-like bismuth molybdate for enhanced photocatalytic activity toward organic pollutant degradation. J. Colloid Interface Sci. 530, 171-178. doi: 10.1016/j.jcis.2018.06.084

Li, S., Jiang, W., Hu, S., Liu, Y., Liu, Y., Xu, K., et al. (2018d). Hierarchical heterostructure of $\mathrm{Bi}_{2} \mathrm{MoO}_{6}$ micro-flowers decorated with $\mathrm{Ag}_{2} \mathrm{CO}_{3}$ nanoparticles for efficient visible-light-driven photocatalytic removal of toxic pollutants. Beilstein J. Nanotechnol. 9, 2297-2305. doi: 10.3762/bjnano.9.214

Li, S., Shen, X., Liu, J., and Zhang, L. (2017a). Synthesis of $\mathrm{Ta}_{3} \mathrm{~N}_{5} / \mathrm{Bi}_{2} \mathrm{MoO}_{6}$ core-shell fiber-shaped heterojunctions as efficient and easily recyclable photocatalysts. Environ. Sci. Nano 4, 1155-1167. doi: 10.1039/C6EN00706F

Li, S. J., Hu, S. W., Jiang, W., Liu, Y., Liu, J. S., and Wang, Z. H. (2017b). Facile synthesis of flower-like $\mathrm{Ag}_{3} \mathrm{VO}_{4} / \mathrm{Bi}_{2} \mathrm{WO}_{6}$ heterojunction with enhanced visible-light photocatalytic activity. J. Colloid Interface Sci. 501, 156-163. doi: 10.1016/j.jcis.2017.04.057

Li, S. J., Hu, S. W., Jiang, W., Liu, Y. P., Zhou, Y. T., Liu, Y., et al. (2018c). Hierarchical architectures of bismuth molybdate nanosheets onto 
nickel titanate nanofibers: facile synthesis and efficient photocatalytic removal of tetracycline hydrochloride. J. Colloid Interface Sci. 521, 42-49. doi: 10.1016/j.jcis.2018.03.033

Li, S. J., Hu, S. W., Jiang, W., and Xu, K. B. (2017c). One-pot solvothermal synthesis of $\mathrm{Ag}$ nanoparticles decorated $\mathrm{BiOCOOH}$ microflowers with enhanced visible light activity. Mater. Lett. 196, 343-346. doi: 10.1016/j.matlet.2017.03.093

Li, S. J., Hu, S. W., Xu, K. B., Jiang, W., Liu, Y., Leng, Z., et al. (2017d). Construction of fiber-shaped silver oxide/tantalum nitride $\mathrm{p}-\mathrm{n}$ heterojunctions as highly efficient visible-light-driven photocatalysts. J. Colloid Interface Sci. 504, 561-569. doi: 10.1016/j.jcis.2017.06.018

Li, S. J., Hu, S. W., Zhang, J. L., Jiang, W., and Liu, J. S. (2017e). Facile synthesis of $\mathrm{Fe}_{2} \mathrm{O}_{3}$ nanoparticles anchored on $\mathrm{Bi}_{2} \mathrm{MoO}_{6}$ microflowers with improved visible light photocatalytic activity. J. Colloid Interface Sci. 497, 93-101. doi: 10.1016/j.jcis.2017.02.069

Li, S. J., Xu, K. B., Hu, S. W., Jiang, W., Zhang, J. L., Liu, J. S., et al. (2017f). Synthesis of flower-like $\mathrm{Ag}_{2} \mathrm{O} / \mathrm{BiOCOOH} \mathrm{p}-\mathrm{n}$ heterojunction with enhanced visible light photocatalytic activity. Appl. Surf. Sci. 397, 95-103. doi: 10.1016/j.apsusc.2016.11.081

Li, X., Yu, J. G., and Jaroniec, M. (2016). Hierarchical photocatalysts Chem. Soc.Rev. 45, 2603-2636. doi: 10.1039/C5CS00838G

Li, Z., Wu, Z., Zhang, S., Shen, J., Feng, W., Du, Y., et al. (2018e). Defect-sate of Indium doped bismuth molybdate nanosheets for enhanced photoreduction of chromium (VI) under visible light illumination. Dalton Trans. 47, 8110-8120. doi: 10.1039/C8DT01807C

Liang, J., Liu, F., Deng, J., Li, M., and Tong, M. (2017). Efficient bacterial inactivation with $\mathrm{Z}$-scheme $\mathrm{AgI} / \mathrm{Bi}_{2} \mathrm{MoO}_{6}$ under visible light irradiation. Water Res. 123, 632-641. doi: 10.1016/j.watres.2017.06.060

Liang, J., Liu, F., Li, M., Liu, W., and Tong, M. (2018). Facile synthesis of magnetic $\mathrm{Fe}_{3} \mathrm{O}_{4} @ \mathrm{BiOI} @ \mathrm{AgI}$ for water decontamination with visible light irradiation: different mechanisms for different organic pollutants degradation and bacterial disinfection. Water Res. 137, 120-129. doi: 10.1016/j.watres.2018.03.027

Lin, W., Yu, X., Zhu, Y., and Zhang, Y. (2018). Graphene oxide/BiOCl nanocomposite films as efficient visible light photocatalysts. Front. Chem. 6:274. doi: 10.3389/fchem.2018.00274

Moroz, P., Boddy, A., and Zamkov, M. (2018). Challenges and prospects of photocatalytic applications based on semiconductor nanocrystals. Front. Chem. 6:353. doi: 10.3389/fchem.2018.00353

Mousavi, M., Habibi-Yangjeh, A., and Pouran, S. R. (2018). Review on magnetically separable graphitic carbon nitride-based nanocomposites as promising visiblelight-driven photocatalysts. J. Mater. Sci. Mater. Electron. 29, 1719-1747. doi: 10.1007/s10854-017-8166-x

Ren, M., Chen, J., Wang, P., Hou, J., Qian, J., Wang, C., et al. (2018). Construction of silver iodide/silver/Bismuth Tantalate Z-scheme photocatalyst for effective visible light degradation of organic pollutants. J. Colloid Interface Sci. 532, 190-200. doi: 10.1016/j.jcis.2018.07.141

Wang, D., and Astruc, D. (2017). The recent development of efficient earthabundant transition-metal nanocatalysts. Chem. Soc. Rev. 46, 816-854. doi: 10.1039/C6CS00629A

Wang, X., Yang, J., Ma, S., Zhao, D., Dai, J., and Zhang, D. (2016). In situ fabrication of $\mathrm{AgIAgVO}_{3}$ nanoribbon composites with enhanced visible photocatalytic activity for redox reactions. Catal. Sci. Technol. 6, 243-253. doi: 10.1039/C5CY00787A

Wang, Y., Wang, H., Xu, A., and Song, Z. (2018). Facile synthesis of $\mathrm{Ag}_{3} \mathrm{PO}_{4}$ modified with GQDs composites with enhanced visible-light photocatalytic activity. J. Mater. Sci. Mater. Electron. 29, 16691-16701. doi: 10.1007/s10854-018-9762-0

Wang, Z. H., Ma, W. H., Chen, C. C., Ji, H. W., and Zhao, J. C. (2011). Probing paramagnetic species in titania-based heterogeneous photocatalysis by electron spin resonance (ESR) spectroscopy-A mini review. Chem. Eng. J. 170, 353-362. doi: 10.1016/j.cej.2010.12.002

Wei, C., Wang, L., Dang, L., Chen, Q., Lu, Q., and Gao, F. (2015). Bottomup-then-up-down route for multi-level construction of hierarchical $\mathrm{Bi}_{2} \mathrm{~S}_{3}$ superstructures with magnetism alteration. Sci. Rep. 5:10599. doi: $10.1038 /$ srep 10599
Wu, M. J., Wu, J. Z., Zhang, J., Chen, H., Zhou, J. Z., Qian, G. R., et al. (2018). A review on fabricating heterostructures from layered double hydroxides for enhanced photocatalytic activities. Catal. Sci. Technol. 8, 1207-1228. doi: 10.1039/C7CY02314F

Wu, Z., Yu, C., Liu, R., Dionysiou, D. D., Yang, K., Wang, C., et al. (2017). Novel fluorinated $\mathrm{Bi}_{2} \mathrm{MoO}_{6}$ nanocrystals for efficient photocatalytic removal of water organic pollutants under different light source illumination. Appl. Catal. B-Environ. 209, 1-11. doi: 10.1016/j.apcatb.2017.02.057

Xiang, Z., Wang, Y., Ju, P., Long, Y., and Zhang, D. (2018). Facile fabrication of $\mathrm{AgI} / \mathrm{BiVO}_{4}$ composites with enhanced visible photocatalytic degradation and antibacterial ability. J. Alloys Compd. 721, 622-627. doi: 10.1016/j.jallcom.2017.06.030

Xiong, J. Y., Cheng, G., Lu, Z., Tang, J. L., Yu, X. L., and Chen, R. (2011). BiOCOOH hierarchical nanostructures: shape-controlled solvothermal synthesis and photocatalytic degradation performances. Cryst. Eng. Comm. 13, 2381-2390. doi: 10.1039/c0ce00705f

Xu, B. Y., An, Y., Liu, Y. Y., Huang, B. B., Qin, X. Y., Zhang, X. Y., et al. (2016). An efficient visible-light photocatalyst made from a nonpolar layered semiconductor by grafting electron-withdrawing organic molecules to its surface. Chem. Commun. 52, 13507-13510. doi: 10.1039/C6CC07849D

Xu, J., Wang, Y., Chen, M., and Teng, F. (2018). A novel BiOCl/BiOCOOH heterojunction photocatalyst with significantly enhanced photocatalytic activity. Mater. Lett. 222, 176-179. doi: 10.1016/j.matlet.2018.04.002

Ye, R., Zhao, J., Wickemeyer, B. B., Toste, F. D., and Somorjai, G. A. (2018). Foundations and strategies of the construction of hybrid catalysts for optimized performances. Nat. Catal. 1, 318-325. doi: 10.1038/s41929-0180052-2

Yuan, X., Wu, Z., Zeng, G., Jiang, L., Zhang, J., Xiong, T., et al. (2018). Synthesis and boosting visible light photoactivity of $\mathrm{Ag@AgI/CdWO} 4$ towards refractory organic pollutants degradation based on interfacialcharge transfer. App. Surf. Sci. 454, 293-304. doi: 10.1016/j.apsusc.2018.05.163

Zhang, G. G., Lan, Z. A., and Wang, X. C. (2016). Conjugated polymers: catalysts for photocatalytic hydrogen evolution. Angew. Chem. Int. Ed. 55, 15712-15727. doi: 10.1002/anie.201607375

Zhang, J. L., and Ma, Z. (2018). Ag- $\mathrm{Ag}_{2} \mathrm{CO}_{3} / \mathrm{Bi}_{2} \mathrm{MoO}_{6}$ composites with enhanced visible-light-driven catalytic activity. J. Taiwan Inst. Chem. Eng. 71, 156-164. doi: 10.1016/j.jtice.2016.11.030

Zhang, L., Zhang, Q., Xie, H., Guo, J., Lyu, H., Li, Y., et al. (2017). Electrospun titania nanofibers segregated by graphene oxide for improved visible light photocatalysis. Appl. Catal. B Environ. 201, 470-478. doi: 10.1016/j.apcatb.2016.08.056

Zhang, M. Y., Shao, C. L., Mu, J. B., Zhang, Z. Y., Guo, Z. C., Zhang, P., et al. (2012). One-dimensional $\mathrm{Bi}_{2} \mathrm{MoO}_{6} / \mathrm{TiO}_{2}$ hierarchical heterostructures with enhanced photocatalytic activity. CrystEngComm 14, 605-612. doi: 10.1039/C1C E05974B

Zhang, W., Sun, Y., Xiao, Z., Li, W., Li, B., Huang, X., et al. (2015). Heterostructures of $\mathrm{CuS}$ nanoparticle/ZnO nanorod arrays on carbon fibers with improved visible and solar light photocatalytic properties. J. Mater. Chem. A 3, 7304-7313. doi: 10.1039/C5TA00560D

Zhu, S. S., and Wang, D. W. (2017). Photocatalysis: basic principles, diverse forms of implementations and emerging scientifc opportunities. Adv. Energy Mater. 7:1700841. doi: 10.1002/aenm.201700841

Conflict of Interest Statement: The authors declare that the research was conducted in the absence of any commercial or financial relationships that could be construed as a potential conflict of interest.

Copyright (c) $2018 \mathrm{Li}$, Jiang, Xu, Hu, Liu, Zhou and Liu. This is an open-access article distributed under the terms of the Creative Commons Attribution License (CC BY). The use, distribution or reproduction in other forums is permitted, provided the original author(s) and the copyright owner(s) are credited and that the original publication in this journal is cited, in accordance with accepted academic practice. No use, distribution or reproduction is permitted which does not comply with these terms. 